\title{
Video Search Technology based on the Motion Characteristics
}

\author{
Yanlin Wang ${ }^{1, \text { a }}$ \\ ${ }^{1}$ Information Engineering College of Wuhan Business University, Wuhan, Hubei, China, 430056 \\ aemail
}

Keywords: Video Search Technology, Motion Characteristics

\begin{abstract}
With the rapid development of computer technology, the emergence of vast amounts of multimedia information on the network, for such a large amount of data, video and other multi-media data has a feature not available - Sports features, so Applied Sport features video search service, for improve the performance of video retrieval system has very important significance.
\end{abstract}

\section{Introduction}

Today's society is the explosive growth of the information society, information technology into every aspect of social life. The development of digital video and audio coding technology, increasing network bandwidth and reduce storage costs, so that people exposed to high levels of multimedia information possible. Among them, the video and audio is the most complex, large digital media form, is the most commonly used form of media. Multimedia information processing and retrieval techniques are mass media organization and management, the use of the key technologies required for editing and interactive services. In terms of management of multimedia data, the ability to automatically retrieve media information is very important. Traditional file name or keyword retrieval based approach is obviously not suitable for video data to the huge amount of data, complex structure. Content-based video retrieval technology is to meet this demand and the rapid development and it is through the visual content of the video data included in the analysis and feature extraction, so that people can use the computer search in line with the subjective experience of similar content segments. To improve the mass media repository search efficiency, the key is to be able to have the means to retrieve based on various characteristics. Content-based video retrieval, the main issues to be addressed include video structure analysis, feature extraction video, description, and expression profiles, video indexing, query matching.

It is generally believed that the visual content of the video data includes information contained in the color, texture, shape and motion, wherein the motion information is different from the video image data unique to the content, for a video clip, the motion video information to reflect changes in the evolution of important information, in order to conduct a comprehensive characterization of video content, motion information is an essential aspect, motion video information in complex and diverse, when the video data is motion analysis, usually formed to move the camera motion information motion information and the main objects in the scene produced separately, called the global motion and local motion, a global movement has strong integrity, a small amount of calculation, the results indicate stability and features convenient features, while local motion is relatively complex calculation Great results not stable enough. In most video sequences, the camera movement is always important to track the movement of people and events in the video, it can be considered global motion information to a certain extent reflects the semantic content of the video, this paper presents a feature that global motion information extracted from the video retrieval technology to provide features not possible with other visual search function.

\section{The Motion Characteristics of Digital Video}

It is generally believed that the visual content of the video data includes information contained in the color, texture, shape and motion, wherein the motion information is different from the video image data-specific content. For a video clip, the motion information is important information to 
reflect changes in the evolution of the video, in order to conduct a comprehensive characterization of video content and motion information is an essential aspect.

Video motion complex and diverse information, when the video data is motion analysis, usually formed by movement of the camera motion information and the motion information generated by the objects in the scene separately, called the global motion and local motion. Global motion with strong integrity, a small amount of calculation, the results indicate stability and features convenient features, while local motion is relatively complex, computationally intensive, the results unstable and difficult to describe with complete features. In most video sequences, the camera movement is always important to track the movement of people and events in the video and it can be considered global motion information to a certain extent reflects the semantic content of the video.

Movement inside the lens includes local motion caused by the motion of an object by the global motion and camera movement caused. Motion camera video will often bring a global impact, such as horizontal movement of the camera makes all pixels are moved horizontally, the focal length of the elongated pixel will diverge from the center to the edges and the focal length will be shortened from the surrounding pixels converging to the center. When only the object movement, much of the background pixels unchanged, and only the moving object and occluded partially changed.

In the video shooting, the camera can be different ways of movement, in order to achieve a particular shot. Motion camera include: pan, turn the lens, the lens moving, sliding lenses. Moving object ever-changing depending on the actual situation, but is an important aspect of video retrieval, especially for video surveillance. For example, a user might need to retrieve an object by moving video clips or video clips of the launch vehicle. Depending on the object motion feature, you can define some object motion events, including: the emergence of (an object appears in the camera) and disappeared (an object disappears from the lens), enter (object motion into the lens) and exit (a movement objects away from the lens), sports (a stationary object begins to move), stop (a moving object is stopped).

Motion analysis features are based on a block matching method, a method based on optical flow equation, the pixel recursive method and Bayesian methods, but the method of calculation of these are very large and very complex to implement. Study on motion characteristics of video data, has been widespread attention, we have made a lot of research, but so far, there are many issues remain unresolved, such as large objects (occupy most of the screen space) and the movement of the lens the movement is difficult to distinguish revealed obscured background and the moving object is difficult to distinguish, especially in the light conditions change, most of the motion analysis method will fail.

\section{Video Retrieval based on the Motion Characteristics}

The video features including visual information can be obtained from each piece of frame image, such as color, texture, shape and spatial relations, as well as sports in addition. Exercise is an essential element of the image sequence analysis, which directly change or movement of the camera linked to the relative position of the spatial entity. Video target and background motion video information as a unique feature, available in a set of parameter values or indicate how spatial relationships change over time string of symbols to represent. Motion video information expressed content development and change in the timeline, it is understood that the description of the video content has a very important role. It is the focus of the content-based video retrieval research.

Video retrieval is often based on the basic characteristics of low-level and high-level semantic features. Low-level features include video content color, texture, shape, motion, etc., characterized by comprising high-level description of the lens, an event and scene semantics. Color, texture feature extraction is mainly carried out by the histogram data of the video frame. Sports features include motion (reflected as the background motion) camera and motion of objects in the scene. Extracts motion feature to the original video data stream, through the pixel basic features and histograms; for compressed video stream can be compressed by the motion vector field data and the like.

Based on a critical step is to retrieve the contents of define a format for describing multimedia 
content, which is stored in the form of information (coding) is also closely related. MPEG-7 is a new generation of audio and video coding international standards and its full name is "Multimedia Content Description Interface." It provides various types of multimedia information in a standardized description of this description and multimedia content itself together and support the user is interested in images, graphics, 3D animation, audio, video, data, fast, efficient retrieval.

Using MPEG-7 video features described are now based on the development trend of video content retrieval and describe how the right is relatively new research questions. Selection of video motion feature extracted using MPEG-7 descriptors to describe, for indexed retrieval on the basis of the description of the MPEG-7. Make full use of standardized description Thought MPEG-7, is conducive to the further application designed for video retrieval system.

\section{The Movement Characteristic Extraction of Video Object}

In the video sequence processing analysis process, since the objectives of the campaign in a video sequence the largest share of the amount of information, often become the segmentation of the video object, so the dynamic segmentation of the video object, a very important step is to distinguish the range of motion and the background. Based on the background is stationary or different situations change, you want to take a different distinction.

Motion Feature Extraction of Camera. Camera movement including turning left and right (Panning), tilt (Tilting), change the focal length (Zooming), the level of tracking (Tracking), vertical track (Booming), front and rear track (Dollying), around the axis of rotation (Rolling), stationary. For the estimated camera motion parameters, computer vision, video encoding and 3D reconstruction of areas has been widely studied. Akutsu et al's method, based on analysis of the Hough space motion vector distribution, which is a method using Hough transform for the parameter space can converge to a point, and parallel lines in good performance. The disadvantage of this approach is that it is a qualitative method, in the case of mixing the camera motion, the motion is not effective for a variety of parameters are determined. Park et al., Used to determine the focal length changes, rotation parameters of the method, by the corresponding data and perspective projection model match. This method can effectively estimate several camera motion parameters, but it does not include the model perspective horizontal track and vertical track two camera motion. Srinivasan et al recommend the use of methods based on optical flow. The advantage of this approach is that almost all cameras can effectively estimate motion parameters.

Motion Activity Feature Extraction. When people watch a video program, you will feel it is slow video sequences, video sequences or fast-paced action sequences. Fast-paced video content including Rally, sports competitions. Slow-paced video content including news announcer lens, interviews lenses. I withdraw the motion activity feature is also based on the motion vectors. According to the recommendations described in MPEG-7 motion activity, the need to extract the intensity of motion activity, activities direction and the spatial distribution characteristics for activities.

Global Motion Feature Extraction. Global motion is global motion video background object contained in the main camera by filming process caused by the movement. Global motion video sequences show the time correlation between, the video features more than other high-level expression of semantic information of the video sequence. By global motion estimation, motion parameters of the model established, is important. Global motion estimation motion model parameters established in the relevant field of image processing and analysis using a wide range, including motion-based segmentation and object tracking and so on. Parametric motion model is also used in MPEG-4, the sprite for compressing and encoding. In MPEG-7, the definition of the parameters of the motion descriptor describes the main characteristics of the global movement. Establish global motion model for video storage and movement-based video retrieval is also important. Global motion estimation of the foreground and background to consider a unified, multi-block according to the video image area of a given interval of time units of analysis, parameter model to show the video movement. Between motion model to define an appropriate similarity measure function can support based on the global motion characteristic of the video 
retrieval, such as retrieving "close to the camera body," "leftward movement of objects" and so on.

Extraction of the Target Track. Feature extraction target trajectory for the object-oriented content-based retrieval has important significance. In the given context, combined with some prior knowledge of the usefulness of the object track is very wide. For example in the monitoring system and if it detects an object trajectory with a certain risk, through the prohibited area, such as speeding, will sound the alarm. In the sports program can be used to determine a specific action, such foul behavior and so on. Object segmentation technology is the key issue of research in recent years, but so far, automatic object segmentation problem has not been solved.

Motion Characteristics Description based on the MPEG-7. Previously used text annotation methods described in the video that the user subjective impact, relatively poor reliability, the use of MPEG-7 video feature can be effective, accurate description. Movement characteristics defined in MPEG-7 descriptors include camera motion descriptor, global motion descriptor, motion activity descriptors, object trajectories descriptor.

\section{Conclusion}

Currently based on the motion characteristics of video retrieval technology is the key technology of digital library construction, motion video feature extraction, effective algorithm will be our further study. Because video data and other data in the form, structure, content and other aspects are different, compared with the images, video structure is more complex, the amount of data is greater, for content-based video retrieval requirements will be higher. Therefore, the application of the motion characteristics of video retrieval services, to improve the performance of video retrieval system has very important significance.

\section{References}

[1] Jia Xinzhang, Li Jingyuan. China Image and Graphics, Vol. 6 (2014) No 53, p.25-26

[2] Peng Sue, Wang Yunhui, Wang Qunyong. Information System, Vol. 12 (2015) No 27, p.74-76

[3] Qian Xiyuan, Jing Jianfen, Hou XuSiem. Computer Science, Vol. 30 (2014) No 19, p.144-145

[4] Wang Kuailiang. Communications and Computer, Vol. 29 (2011) No 27, p.21-23

[5] Zhang Gongxu, Sun Jing. Information System, Vol. 8 (2013) No 27, p.57-60 